\title{
Nanoporous silicon-based surface patterns fabricated by UV laser interference techniques for biological applications
}

This content has been downloaded from IOPscience. Please scroll down to see the full text.

2016 J. Phys. D: Appl. Phys. 49225401

(http://iopscience.iop.org/0022-3727/49/22/225401)

View the table of contents for this issue, or go to the journal homepage for more

Download details:

This content was downloaded by: lornawroe

IP Address: 89.202.245.164

This content was downloaded on 10/03/2017 at 13:11

Please note that terms and conditions apply.

You may also be interested in:

Chemical stabilization of porous silicon for enhanced biofunctionalization with immunoglobulin Nelson Naveas, Vicente Torres Costa, Dario Gallach et al.

Fabrication of silicon nanowire arrays by near-field laser ablation and metal-assisted chemical etching

D Brodoceanu, H Z Alhmoud, R Elnathan et al.

Porous silicon photonic devices using pulsed anodic etching of lightly doped silicon

J Escorcia-Garcia, O Sarracino Martínez, J M Gracia-Jiménez et al.

Laser applications in nanotechnology: nanofabrication using laser ablation and laser

nanolithography

G N Makarov

Single-step fabrication of microfluidic channels filled with nanofibrous membrane using femtosecond laser irradiation

Amirhossein Tavangar, Bo Tan and K Venkatakrishnan

One dimensional Si/Ge nanowires and their heterostructures for multifunctional applications-a

review

Samit K Ray, Ajit K Katiyar and Arup K Raychaudhuri

CVD of polymeric thin films: applications in sensors, biotechnology, microelectronics/organic electronics, microfluidics, MEMS, composites and membranes

Gozde Ozaydin-Ince, Anna Maria Coclite and Karen K Gleason

Period dependence of laser induced patterns in metal films

R J Peláez, C N Afonso, M Škere et al. 


\title{
Nanoporous silicon-based surface patterns fabricated by UV laser interference techniques for biological applications
}

\author{
G Recio-Sánchez ${ }^{1,5}$, R J Peláez ${ }^{2}$, F Vega $^{3}$ and R J Martín-Palma ${ }^{4}$ \\ ${ }^{1}$ Núcleo de Energías Renovables, Departamento de Ciencias Matemáticas y Físicas, Universidad Católica \\ de Temuco, Reducindo Ortega 02950, Temuco, Chile \\ 2 Laser Processing Group, Instituto de Óptica, Consejo Superior de Investigaciones Científicas, serrano \\ 121, 28006, Madrid, Spain \\ 3 Departament d'Óptica i Optometría, Universidad Politécnica de Cataluña, BarcelonaTECH, Violinista \\ Vellsolá 37, 08222, Terrasa, Spain \\ ${ }^{4}$ Departamento de Física Aplicada, Universidad Autónoma de Madrid, Cantoblanco, 28049 Madrid, \\ Spain \\ E-mail: grecio@uct.cl
}

Received 7 January 2016, revised 17 March 2016

Accepted for publication 1 April 2016

Published 4 May 2016

\begin{abstract}
The fabrication of selectively functionalized micropatterns based on nanostructured porous silicon (nanoPS) by phase mask ultraviolet laser interference is presented here. This singlestep process constitutes a flexible method for the fabrication of surface patterns with tailored properties. These surface patterns consist of alternate regions of almost untransformed nanoPS and areas where nanoPS is transformed into Si nanoparticles (Si NPs) as a result of the laser irradiation process. The size of the transformed areas as well as the diameter of the Si NPs can be straightforwardly tailored by controlling the main fabrications parameters including the porosity of the nanoPS layers, the laser interference period areas, and laser fluence. The surface patterns have been found to be appropriate candidates for the development of selectively-functionalized surfaces for biological applications mainly due to the biocompatibility of the untransformed nanoPS regions.
\end{abstract}

Keywords: biomaterials, laser interference, micropattern

\section{Introduction}

Nanostructured porous silicon (nanoPS) can be regarded as a somewhat complex network of silicon nanocrystals embedded into a porous matrix [1]. This nanomaterial is generally fabricated by the electrochemical etching of silicon wafers in hydrofluoric acid (HF)-based solutions. When crystalline silicon is transformed into nanoPS during the anodization process, quantum confinement effects (QCEs) manifest as a consequence of the reduction in size of the Si crystals [2]. This process also leads to several other novel physico-chemical properties. In fact, the efficient photoluminescence and electroluminescence in the

\footnotetext{
${ }^{5}$ Author to whom any correspondence should be addressed.
}

visible range at room temperature are commonly attributed to QCEs $[3,4]$. In addition, the tunable optical and structural properties of nanoPS, together with its high surface reactivity which opens the possibility of transforming it into a biocompatible material, make nanoPS an appropriate nanostructured material for the development of a wide variety of devices in a broad range of fields including electronics, photonics, telecommunications, and biomedicine [5-7].

Surface micro- and nanopatterning are becoming important techniques to enhance the performance of 'traditional' materials. In the case of nanoPS, different patterns have been developed for such applications as photonic devices, diffraction gratings, high-sensitivity biosensors, etc [8-10]. In order to fabricate these patterns, various techniques such as dry soft 
lithography [11], press stamp [12], ion bombardment [13], or microstructuring crystalline silicon before electrochemical etching [14] have been used. One of the most used and precise technique is the combination of focused high-energy proton beams and electrochemical etching [15]. In this particular case, the selective formation of nanoPS allows the development of a wide variety of photonic devices including waveguides and photonic crystals $[16,17]$. In addition, due to the biocompatibility of Si and nanoPS, these patterns have been also used for the fabrication of tailored platforms for cell adhesion $[18,19]$. However, none of these methods have the capability to offer flexibility in the pattern design in a timeefficient process, over large areas, and in a single-step process.

In this context, the present work reports on the fabrication of 1D- and 2D surface patterns based on nanoPS by phase mask ultraviolet laser interference. This is a single-step and flexible approach which allows producing an ample variety of patterns consisting in defined regions of almost untransformed nanoPS and areas where nanoPS has been transformed into Si nanoparticles (Si NPs). Furthermore, by controlling the main parameters in the fabrication process such as nanoPS porosity and laser fluence, the key features of the patterns, including the period and the diameter of the Si NPs, can be precisely tailored. In addition, the production of selectively functionalized surfaces from these patterns is presented.

\section{Experimental}

\section{Nanostructured porous silicon fabrication}

NanoPS layers were fabricated by electrochemical etching low resistivity $p$-type silicon wafers $(\rho=0.01-0.05 \Omega \cdot \mathrm{cm}$; orientation <1 $00>$ ) in HF (48 wt.\%):ethanol (98 wt.\%) (1:2) solutions. The current density was adjusted between 5 and $80 \mathrm{~mA} \mathrm{~cm}{ }^{-2}$ in order to vary the porosity of the layers. The etching time was also changed between 10 and $45 \mathrm{~s}$ to control the thickness of the layers. In this work two different types of nanoPS layers with very different properties were selected for their detailed analysis. These were named 'nanoPS-LP', i.e. low porosity nanoPS layers which were fabricated by applying a current density of $5 \mathrm{~mA} \mathrm{~cm} \mathrm{~cm}^{-2}$ for $45 \mathrm{~s}$ and 'nanoPS-HP', or high porosity nanoPS layers, which were synthesized under constant applied current of $80 \mathrm{~mA} \mathrm{~cm}^{-2}$ for $10 \mathrm{~s}$.

\section{Functionalization process}

After the electrochemical etching process the surface of nanoPS is $\mathrm{Si}_{\mathrm{x}} \mathrm{H}_{\mathrm{x}}$ terminated [20]. Accordingly, aiming at stabilizing its relatively high chemical activity, the nanoPS layers were immersed in $\mathrm{H}_{2} \mathrm{O}_{2}(30 \% \mathrm{v} / \mathrm{v})$ for 90 min and subsequently rinsed in absolute ethanol. Surface functionalization was performed by the immersion of the nanoPS in aminopropyltriethoxysilane (APTS):toluene (2:1000) solutions for $15 \mathrm{~min}$. In this process, the presence of $\mathrm{SiO}_{2}$ on the surface of nanoPS (formed by the oxidation as a result of stabilization process), reacts with the APTS-based solution leading to a large surface density of amino groups [21]. It is worth mentioning that amino groups were selected for this study given that they are present in proteins and their chemical interaction with other functional groups is fairly well understood.

\section{Fabrication of tailored surface patterns by UV interference}

Tailored surface patterns based on nanoPS were fabricated by an interferential process where single pulses of an excimer laser $(\lambda=193 \mathrm{~nm}$ and $\tau=20 \mathrm{~ns})$ were used to expose a fringe phase mask optimized for high efficiency in the \pm 1 diffraction orders. These orders are made to overlap and interfere at the surface of nanoPS layers by means of two lenses in telescope configuration. Thus, the nanoPS surface is exposed to a modulated intensity formed by the maxima and minima of interference. By using different configuration for the lenses, the period of the pattern can easily be modified. In addition, by combining different laser pulses or diverse phase masks, 2D patterns can be obtained. Further details can be found elsewhere [22].

\section{Characterization}

Optical characterization was carried out with an UV - vis spectrophotometer (Jasco V-560) equipped with a Hamamatsu R928 photomultiplier. All reflectance measurements were performed in the 400 to $800 \mathrm{~nm}$ range with a $2 \mathrm{~nm}$ interval and with $1 \mathrm{~s}$ integration time.

Morphological characterization of nanoPS layers and surface patterns was carried out by using a field emission scanning electron microscopy (FESEM) XL 30S-FEG (Philips) in planar (PV) and cross-sectional (CS) views. No metallization was required to characterize the samples. The effectiveness of the functionalization process of the nanoPS surfaces with APTS was evaluated using fluorescein isothiocyanate (FITC, Sigma). FITC is a fluorescent molecular probe which reacts covalently with the amino group of APTS and was visualized in a fluorescence inverted microscope (Olympus IX81, Olympus Corporation, Shinjuku, Tokyo, Japan) coupled to a CCD color camera.

\section{Result and discussion}

The porosity, $P$, of the two different types of nanoPS layers studied in this work (see experimental section) was determined by gravimetry using the following relationship [2]:

$$
P(\%)=\frac{m_{1}-m_{2}}{m_{1}-m_{3}} \cdot 100
$$

$m_{1}$ being the silicon mass before the electrochemical etching process, $m_{2}$ the mass after the nanoPS layer is formed, and $m_{3}$ the mass remaining upon removal of the nanoPS layer in alkaline solutions. The average porosity (mean and standard deviation of 10 measurements per each type of layer) was found to be $42 \pm 8 \%$ and $72 \pm 5 \%$. From now on, these samples will be referred to as low porosity (nanoPS-LP) and high porosity (nanoPS-HP) respectively.

Figure 1 shows PV and CS FESEM images of two representative nanoPS-LP (figures 1(a) and (c)) and nanoPS-HP (figures 1(b) and (d)) layers before laser processing. It was 
determined by image analysis that the nanoPS-LP layers have an average pore size of $20 \mathrm{~nm}$ in diameter and a surface pore density of $38 \pm 1 \%$, whereas nanoPS-HP layers show an average pore diameter of $50 \mathrm{~nm}$ and a surface pore density of $68 \pm 1 \%$. These parameters are in good agreement with the porosities determined by gravimetry. In fact, in both cases the surface porosity is smaller than bulk porosity, an effect already reported for nanoPS. Additionally, CS FESEM images show that the nanoPS layers have a thickness around $500 \mathrm{~nm}$ in both cases. Besides, the pore structure can be described as an interlaced pore network in the case of nanoPS-LP and as an array of longitudinal pores in the case of nanoPS-HP.

Figure 2(a) shows the experimental reflectance spectra of the two different layers (nanoPS-LP and nanoPS-HP) of similar thickness $(\sim 500 \mathrm{~nm})$ and that of crystalline silicon as a reference. Typical thin film interferences are observed in the reflectance spectra of both porous layers. As expected, increased light reflection towards the blue part of the spectra (shorter wavelengths) is manifested in nanoPS-LP. As we will discuss below, this behavior has a significant effect in the morphology of the layers upon laser irradiation. From these spectra, the refractive index in the visible range of both layers as a function of the wavelength was calculated making use of a mathematical algorithm previously developed by our research group [23]. The resulting refractive indexes as a function of the wavelength are represented in figure 2(b).

Figure 3 shows 1D patterns fabricated on nanoPS-LP (figure 3(a)) and nanoPS-HP layers (figure 3(b)) by the incidence of one laser pulse, setting the laser fluence to $43 \mathrm{~mJ} \mathrm{~cm} \mathrm{~cm}^{-2}$ and a combination of projection lenses to obtain periods of $6,3 \mu \mathrm{m}$. The resulting patterns consist of alternating areas of almost unaltered nanoPS (dark contrast areas in figures 3(a) and (b)) and regions where nanoPS has been altered as a result of the irradiation process (bright contrast areas in figures 3(a) and (b)). Figures 3(c) and (d) show a magnified view of the irradiated fringes for the nanoPS-LP and nanoPS-HP layers respectively. In the case of the nanoPS-LP layers, the images indicate that the laser irradiation process has led to a partial melt-down of the nanoPS layers and, as a result, in these zones a reduction of the average pore density of the layers and an increase of the average pore diameter is observed. In contrast, in the case of the nanoPS-HP layers the laser irradiation process induces a more notorious transformation, in which the melted nanoPS layer is transformed into Si NPs with an average diameter of $120 \mathrm{~nm}$ and an ample diversity of shapes. It is noticeable that the average diameter of the Si NPs as well as the percentage of transformed region can be controlled in the cases of the nanoPS-HP layers by selecting the appropriate laser fluence [24].

In addition, slightly tilted CS FESEM images of the patterns are shown in figures 4(a) and (b) for nanoPS-LP and nanoPS-HP respectively. In the case of the nanoPS-LP layers, the CS image shows that the transformed regions in the pattern modify slightly their thickness. This might indicate that the irradiation process must have induced a shallow melting of the layers in the regions exposed to the laser intensity maxima. As a consequence, the transformation on these regions is restricted to the surface of the porous layer.
However, in the case of the nanoPS-HP layers a depth topographic pattern with a modulated shape is generated upon irradiation. In this case, the pattern is composed of alternate regions of untransformed (those exposed to the laser intensity minima) and transformed nanoPS (those that were irradiated with the laser intensity maxima). In particular, in the region of highest laser intensity (figure 4(d)), the thickness of the original porous layer $(500 \mathrm{~nm})$ is reduced to $250 \mathrm{~nm}$. Also, on top of the remnant nanoPS layer, Si NPs with a typical round shape are observed.

The different patterns observed for the two types of nanoPS layers upon irradiation with the same fluence and laser intensity profile, i.e. shallow transformed regions with reduced pore density and increased pore diameter for nanoPSLP and alternate modulated regions with in-depth reduction of the thickness and formation of Si NPs for nanoPS-HP, can be associated to three main factors. First, the higher reflection of the nanoPS-LP layers in the UV range which reduces the power density of the layer that is absorbed. Second, the thermal conductivity from nanoPS layer which is reduced for higher porosities [25]. In this case, the lower thermal conductivity of nanoPS-HP favors the increase of temperature in the irradiate regions, reaching the melting temperature faster and allowing the melt depth going further in-depth. Third, the smaller density of the nanoPS-HP layer gives advantage to the formation of bigger and well isolated Si NPs, whereas the higher density of the nanoPS-LP promote the coalescence of neighbor molten NPs and herein the percolation threshold is reached easily and the formation of big NPs is truncated. As discussed below, the production of biofunctionalized patterns on nanoPS relies on the formation of regions with very different physico-chemical and morphological properties, which was not accomplished using nanoPS-LP samples. In contrast, alternate modulated regions with in-depth reduction of the thickness and formation of Si NPs were produced on nanoPS-HP. Accordingly, nanoPS-LP layers were not used for the biofunctionalization processes.

In order to prove the versatility of the laser technique to produce different patterns on nanoPS surfaces, figure 5 shows PV SEM images of several other patterns fabricated on nanoPS-HP layers. Figures 5(a) and (b) show 1D patterns but with different periods, $1.7 \mu \mathrm{m}$ and $31 \mu \mathrm{m}$ respectively. The period of the pattern can be changed just by adjusting the configuration lenses which produce the laser interference.

In addition, figures 5(c) and (d) show two different 2D patterns. Figure 5(c) shows pattern consisting in a square lattice grid of irradiated zones and almost untransformed zones. This pattern was fabricated by two pulses of laser, rotating $90^{\circ}$ the sample between pulses. Figure 5(d) shows a 2D oblique lattice. To fabricate this pattern, a pair of phase masks with $90^{\circ}$ relative orientation is required to modulate the laser beam so as to obtain a $2 \mathrm{D}$ irradiation pattern. As it was the case of the $1 \mathrm{D}$ patterns, the period of the two different $2 \mathrm{D}$ patterns can be controlled by adjusting the configuration of the projection lenses.

As a proof of concept, the various laser fabricated patterns have been tested as selective biofunctionalization platforms. The biocompatibility of nanoPS has been extensively studied due to its many potential applications in the broad field of 
a)

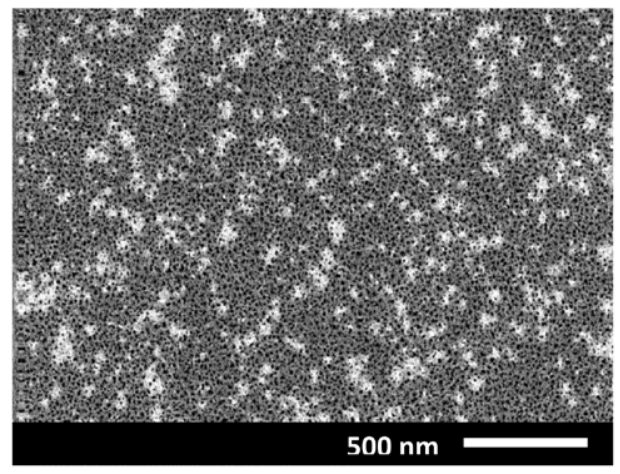

c)

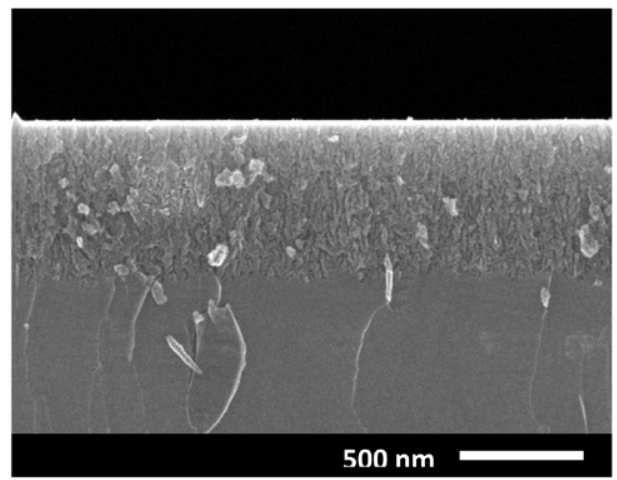

b)

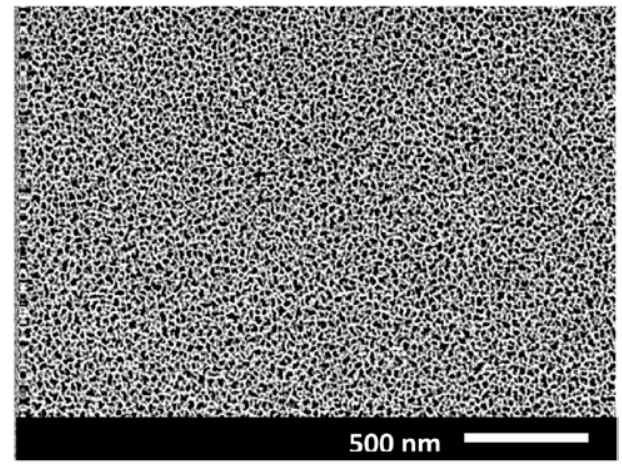

d)

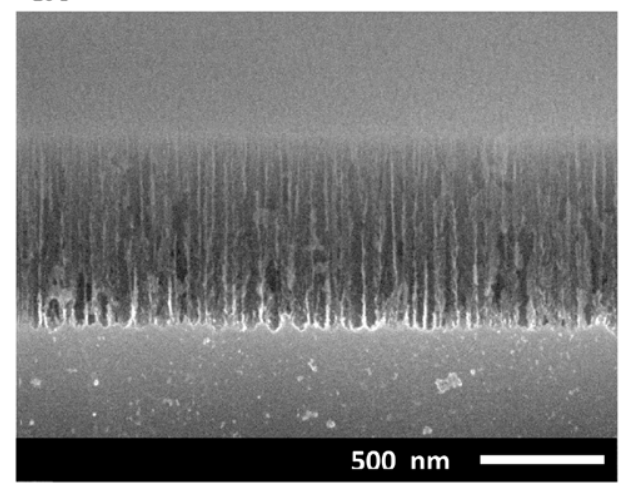

Figure 1. FESEM planar view (PV) images of the surface of (a) nanoPS-LP and (b) nanoPS-HP as formed. (c) Cross sectional (CS) images of characteristic nanoPS-LP and (d) nanoPS-HP layers. NanoPS-LP was formed using an applied current density of $5 \mathrm{~mA} \mathrm{~cm}-2$ for $45 \mathrm{~s}$ and nanoPS-HP was formed under an applied current density of $80 \mathrm{~mA} \mathrm{~cm}^{-2}$ for $10 \mathrm{~s}$.
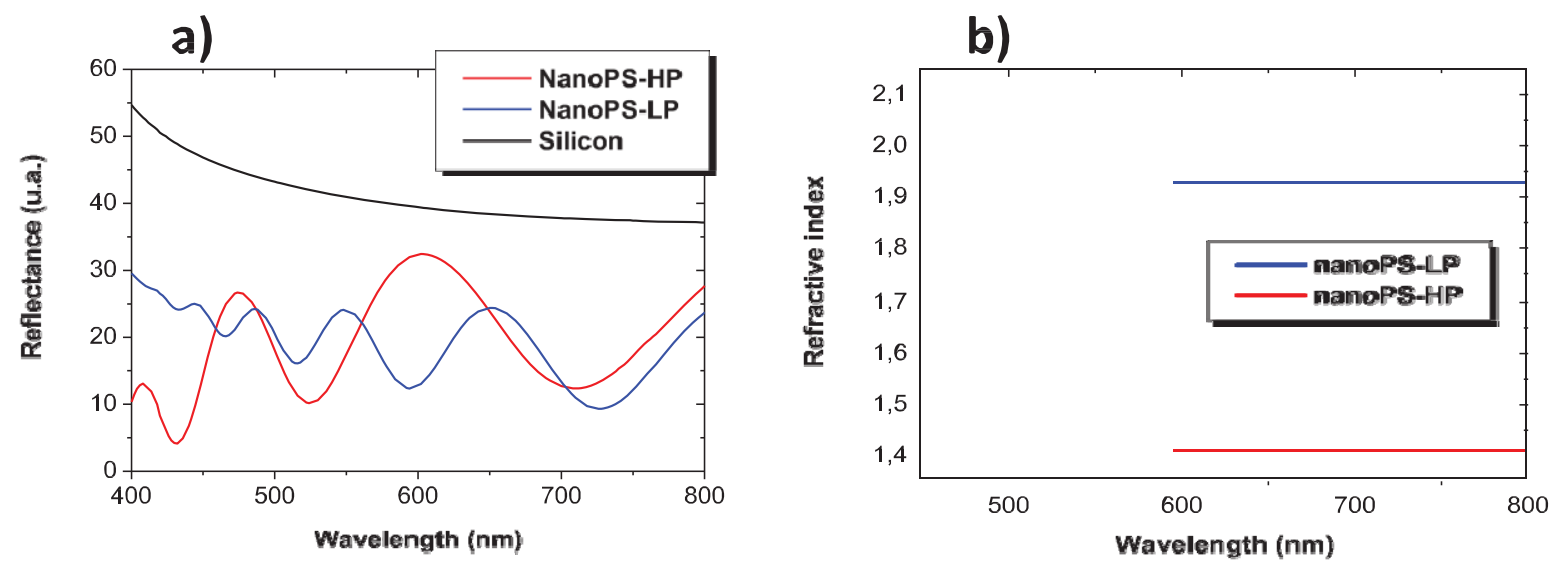

Figure 2. (a) Experimental reflectance spectra of silicon reference (black line), nanoPS-LP layer (blue line) and nanoPS-HP layer (red line). (b) Refractive index of nanoPS layer calculated from reflectance spectra [23].

biomedicine (see for example [26]). Figure 6(a) shows a fluorescence microscope image of nanoPS biofunctionalized with APTS. The surface of nanoPS is biofunctionalized by immersion into a APTS:toluene $(2: 1000)$ solutions after a previous stabilization process (immersion in $\mathrm{H}_{2} \mathrm{O}_{2}$ solutions for $90 \mathrm{~min}$, as described above). In order to be able to assess the efficiency of the biofunctionalization process by fluorescence microcopy, FITC, which is known to specifically react with amino groups, was used. As shown in figure 6(a), the biofunctionalization process results in an uniform fluorescence intensity across the image proving that homogenous biofunctionalized layers are produced on top of the surface of nanoPS. Following this process, amino groups are bonded on the surface of nanoPS by a previous oxidation of the surface, remaining intact most of the $\mathrm{O}-\mathrm{Si}-\mathrm{CH}_{2}-\mathrm{CH}_{2}-\mathrm{CH}_{2}-\mathrm{NH}_{2}$ groups present in APTS molecules [27, 28]. 


\section{a)}

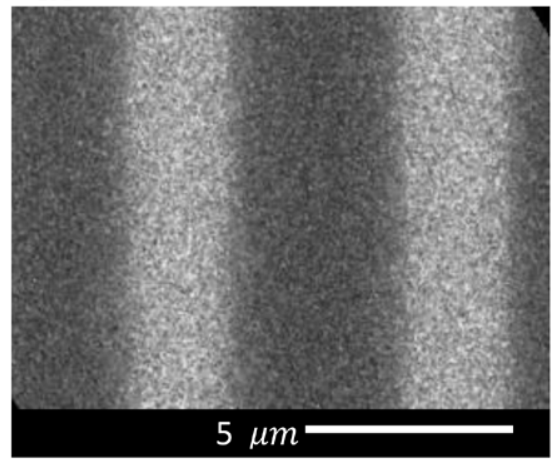

c)

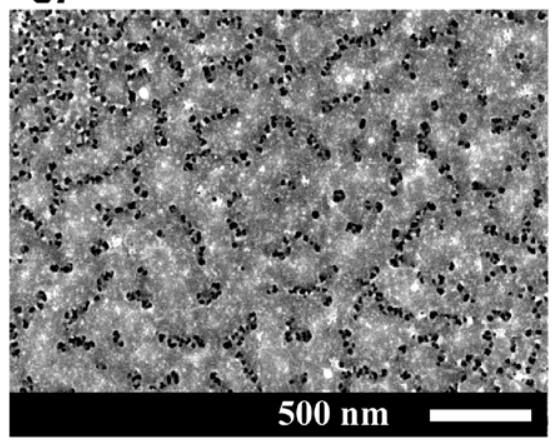

b)

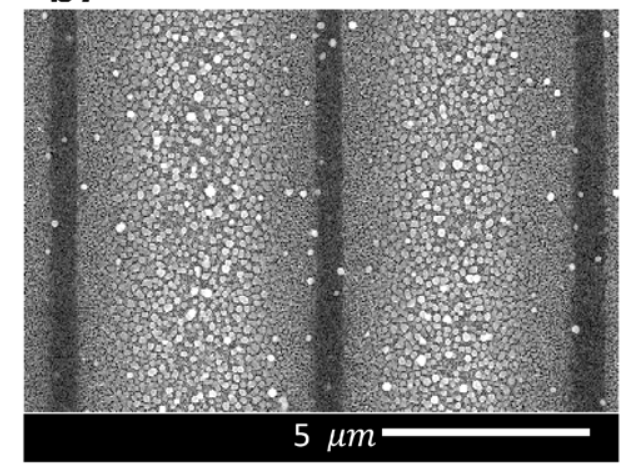

\section{d)}

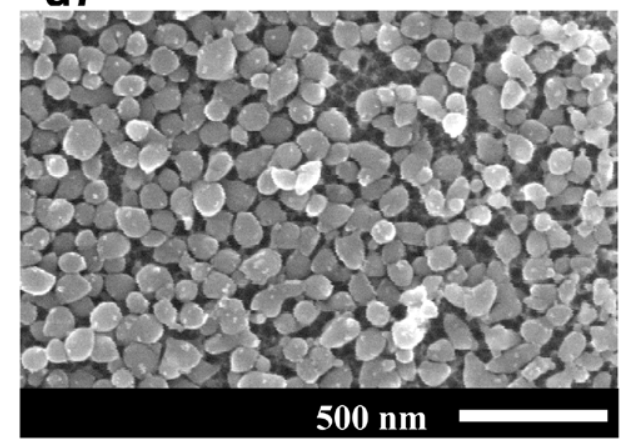

Figure 3. FESEM planar view images of $1 \mathrm{D}$ patterns formed with a laser fluence of $43 \mathrm{~mJ} \mathrm{~cm}{ }^{-2}$ and a period of $6.3 \mu \mathrm{m}$ on (a) nanoPS-LP and (b) nanoPS-HP layers. Magnified views of the transformed zones for (c) nanoPS-LP and (d) nanoPS-HP layers.
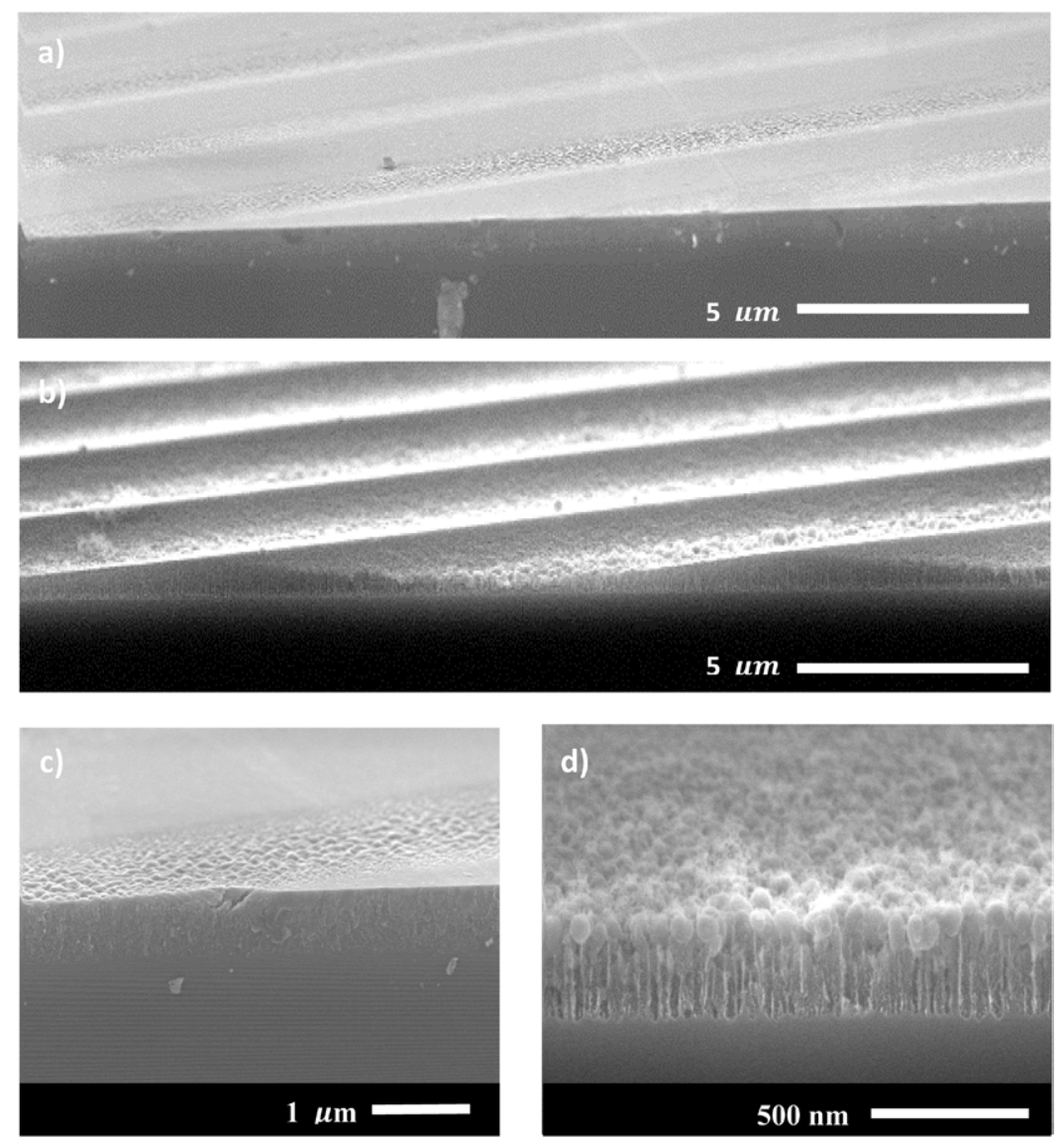

Figure 4. Slightly tilted cross section FESEM images of the patterns formed on (a) nanoPS-LP layer and (b) nanoPS-HP layer. Magnified views of the transformed zones are shown in (c) for nanoPS-LP and (d) nanoPS-HP layer. 
a)

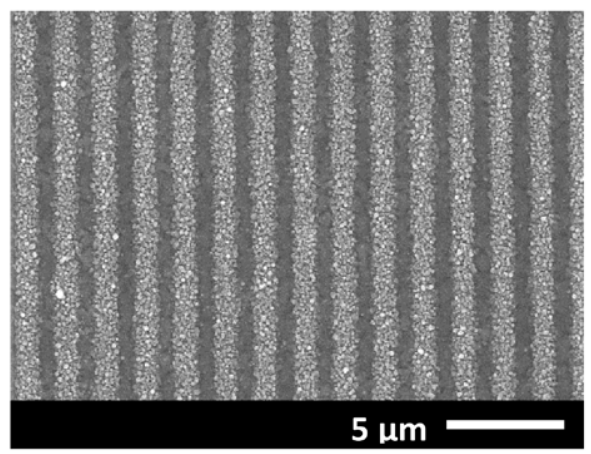

c)

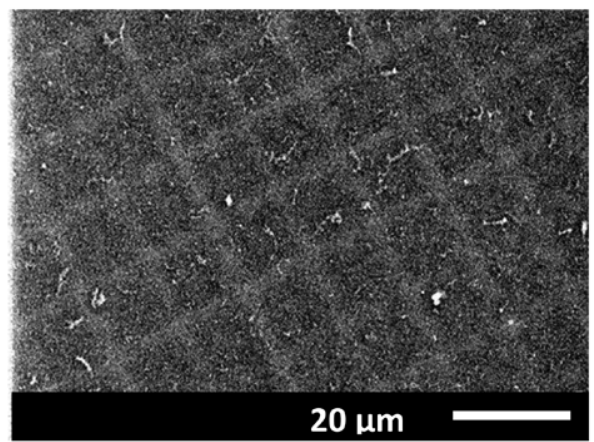

b)

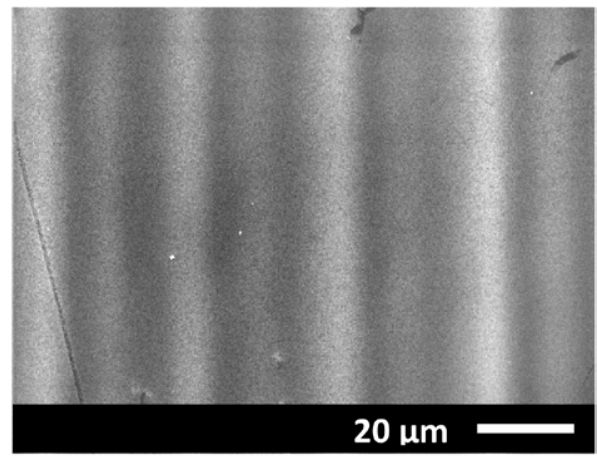

d)

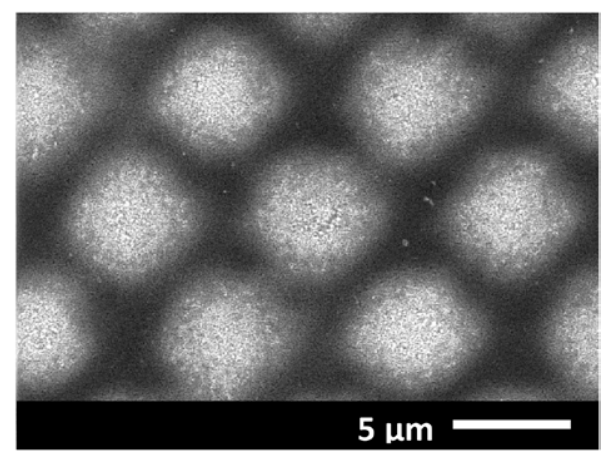

Figure 5. FESEM images of pattern fabricated by interference laser irradiation on nanoPS layers. (a) 1D pattern of $1.7 \mu \mathrm{m}$ of period. (b) $1 \mathrm{D}$ pattern with a period of $31 \mu \mathrm{m}$. (c) 2D pattern based on a square mesh. (d) 2D pattern with a hexagonal lattice.

a)

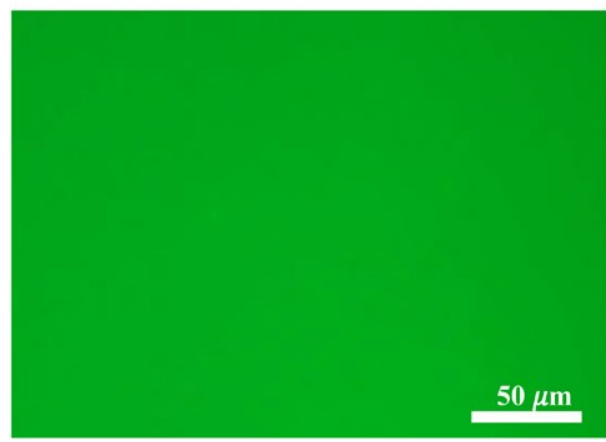

b)

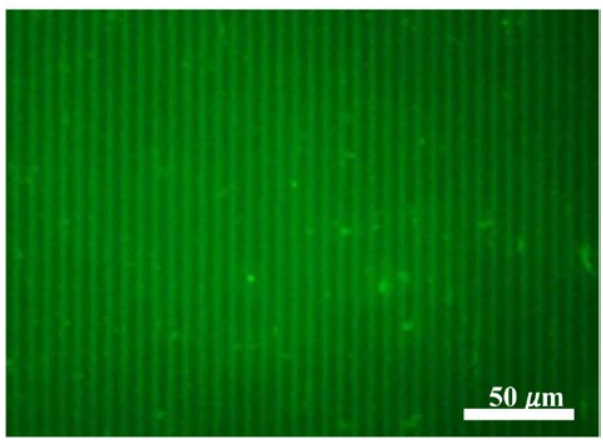

Figure 6. Fluorescence microscope images of nanoPS surface biofunctionalizated with APTS (a) before laser irradiation and (b) after interference laser irradiation.

To create selective biofunctionalized platforms, direct laser writing has been previously used [29]. Since laser irradiation alters the properties of the biofunctionalized layers, selective biofunctionalized platforms can only be obtained from homogeneous surfaces by means of an 'on and off' irradiation process, i.e. alternating irradiated and not irradiated regions. However, by phase mask laser interference, the same platforms can be fabricated with a single shot in a time efficient process (ns range) and over relatively large areas $\left(\mathrm{mm}^{2}\right)$. Figure $6(\mathrm{~b})$ shows a fluorescence image of the platform fabricated after exposure to one laser pulse with a fluence $45 \mathrm{~mJ} \mathrm{~cm}^{-2}$ and a period of $6.3 \mu \mathrm{m}$ to the homogeneous APTS-biofunctionalized nanoPS-HP layer shown in figure 6(a). The resulting platform is based on regions where the laser irradiation has altered the biofunctionalization layer (dark fringes), and untransformed biofunctionalizated fringes (lighter areas). As a consequence, chemical interaction with other functional groups would preferentially occur at the biofunctionalizated fringes.

Although figure 6(b) indicates that in the regions exposed to the laser intensity minima the biofunctionalized layer remains almost untransformed, it would be desirable that the process of patterning the biofunctionalized platforms were carried out in such a way that the biofunctionalization layer were not irradiated by the laser beam. For this purpose, we have first fabricated by phase mask UV laser interference $1 \mathrm{D}$ patterns on fresh nanoPS-HP layers and, eventually, these patterns were subjected to the biofunctionalization process. Figures 7(a) and (b) show two different selective biofunctionalized platforms 


\section{a)}

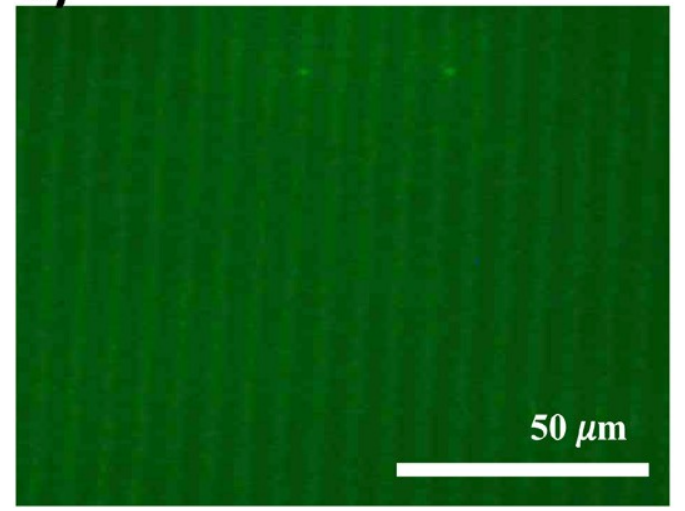

b)

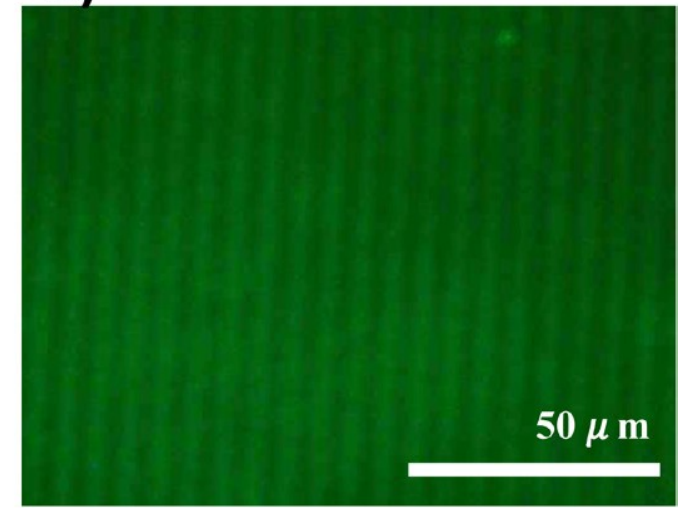

Figure 7. 1D selective biofunctionalizated platforms with a period of $6.3 \mu \mathrm{m}$ based on 1D nanoPS pattern fabricated by laser interference with different laser fluence; (a) $45 \mathrm{~mJ} \mathrm{~cm}^{-2}$ and (b) $22 \mathrm{~mJ} \mathrm{~cm}^{-2}$.

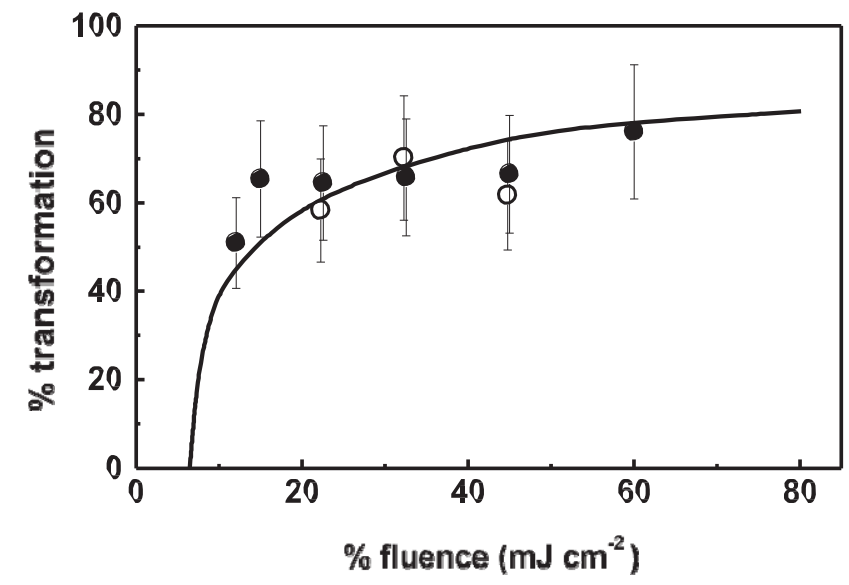

Figure 8. Percentage of non-biofunctionalized region normalized to the period, for samples that have been biofunctionalized before $(\bullet)$ and after (o) laser irradiation. The continuous line represent the percentage of meelted region calculated using the heat flow equation (see [24] for details).

fabricated by the aforementioned process. The patterns were fabricated by exposing to one interference laser pulse with different laser fluence $\left(45 \mathrm{~mJ} \mathrm{~cm}^{-2}\right.$ and $22 \mathrm{~mJ} \mathrm{~cm}^{-2}$ for figures 7(a) and (b), respectively) on nanoPS-HP layers, fixing the period at $6.3 \mu \mathrm{m}$. After the fabrication of the patterns, these were subjected to the same biofunctionalization process with APTS.

As shown in figures 7, the platforms are also formed by efficient biofunctionalization fringes (lighter areas) and regions in which the biofunctionalization process did not produce an efficient homogeneous biofunctionalized layer (darker areas). In this occasion, the different biocompatibility between untransformed nanoPS surface and Si NPs obtained after laser irradiation allows the development of these of platforms. The higher surface chemical reactivity of the nanoPS regions in comparison to the ones with SiNPs makes easy the bonding of $\mathrm{O}-\mathrm{Si}-\mathrm{CH}_{2}-\mathrm{CH}_{2}-\mathrm{CH}_{2}-\mathrm{NH}_{2}$ groups present in APTS molecules. In addition, the higher surface area of the nanoPS layers along with their porous structure allow to obtain homogeneous biofunctionalized layers by the process described below.

Finally, a series of fringes with fluencies in the $12-60 \mathrm{~mJ}$ $\mathrm{cm}^{-2}$ range where produced by the two process described above, i.e. after and before the biofuncionalization process. Fluorescence images show that by changing the laser fluence, the width of the biofunctionalized fringes can be selectively tailored. Average profiles of the fluorescence signal along the period have been obtained at different fluences and the regions with maximal intensity, related with the biofunctionalized fringes have been obtained. Figure 8 shows the estimated relative non-biofunctionalized width along the period for the different fringes analyzed, where the scale bars are related with the quality of the fluorescence images. Fringes biofuncionalized after and before laser irradiation have a similar behavior; biofuncionalized regions decrease in the 50 to $25 \%$ range as the fluence increase from 12 to $60 \mathrm{~mJ} \mathrm{~cm}^{-2}$ and tend to saturate for high fluences. In addition, the calculation of the percentage of melted regions in the total irradiated area obtained as described in [24] has been included in figure 8 . Simulation of melted and non-biofuncionalized regions are in good agreement, within the experimental error. This suggests that the morphology of the surface of nanoPS-HP is a key parameter since it determines the fluorescence intensity and thus the adhesion and activity of the biofunctionalized regions. Accordingly, it can be affirmed that these selective biofunctionalized platforms are promising candidates for novel biological and biomedical applications.

\section{Conclusions}

Phase mask UV laser interference has been demonstrated as a versatile and promising technique for the fabrication of 1D- and 2D patterns on nanoPS in an efficient time process (ns range) and over relatively large areas $\left(\mathrm{mm}^{2}\right)$. The patterns generated upon laser interference are composed by nanoPS areas and regions where nanoPS layer has melted and transformed into Si NPs for high porosity layers. By controlling the main parameters in the fabrication process such as porosity of nanoPS layer, laser fluence or projection lenses configuration, the principal characteristic of the patterns can be determined including the width of transformed regions, period of the patterns and even the diameter of the Si NPs.

Due to the high reactivity of nanoPS which is unaltered in the regions irradiated with lower intensity and allows 
nanoPS become in a biocompatible material, selective biofunctionalization platforms can be obtained from these patterns. Percentage of biofunctionalized region, motive and size of periodic patterns can be tailored by the laser parameters. In this way, these platforms are ideal candidates for the development of novel biological and biomedical applications.

\section{Acknowledgments}

The authors gratefully acknowledge funding from Ministerio de Economía y Competitividad (Spain) under research projects MAT2013-46572-C2-1-R and MAT2014-54826-C2-1-R. R P acknowledges the grant JCI-2012_13034 from the Juan de la Cierva program.

\section{References}

[1] Martín-Palma R J, Pascual L, Herrero P and Martínez-Duart J M 2002 Direct determination of grain sizes, lattice parameters and mismatch of porous silicon Appl. Phys. Lett. 81 25-7

[2] Bisi O, Ossicini S and Pavesi L 2000 Porous silicon: a quantum sponge structure for silicon based optoelectronics Surf. Sci. Rep. 38 1-126

[3] Canham L T 1990 Silicon quantum wire array fabrication by electrochemical and chemical dissolution of wafers Appl. Phys. Lett. 57 1046-8

[4] Koshida N and Koyama H 1992 Visible electroluminescence from porous silicon Appl. Phys. Lett. 60 347-9

[5] Torres-Costa V and Martin-Palma R J 2010 Application of nanostructured porous silicon in the field of optics. A review J. Mater. Sci. 45 2823-38

[6] Martin-Palma R J, Manso-Silvan M and Torres-Costa V 2010 Biomedical applications of nanostructured porous silicon: a review J. Nanophotonics 4042502

[7] Gautier G and Leduc P 2014 Porous silicon for electrical isolation in radio frequency devices: a review Appl. Phys. Rev. 1011101

[8] Birner A, Wehrspohn R B, Gösele U M and Busch K 2001 Silicon-based photonic crystals Adv. Mater. 13 377-88

[9] Lai M, Sridharan G M, Parish G, Bhattacharya S and Keating A 2012 Multilayer porous silicon diffraction gratings operating in the infrared Nanoscale Res. Lett. $71-8$

[10] Flavel B S, Sweetman M J, Shearer C J, Shapter J G and Voelcker N H 2011 Micropatterned arrays of porous silicon: toward sensory biointerfaces ACS Appl. Mater. Interfaces $32463-71$

[11] Gargas D J, Muresan O, Sirbuly D J and Buratto S K 2006 Micropatterned porous-silicon Bragg mirrors by dryremoval soft lithography $A d v$. Mater. 183164

[12] Ryckman J D, Jiao Y and Weiss S M 2013 Three-dimensional patterning and morphological control of porous nanomaterials by gray-scale direct imprinting Sci. Rep. 31502

[13] Recio-Sánchez G, Torres-Costa V, Manso-Silván M and Martín-Palma R J 2012 Nanostructured porous silicon photonic crystal for applications in the infrared J. Nanotechnol. 2012106170

[14] Müller F, Birner A, Gösele U, Lehmann V, Ottow S and Föll H 2000 Structuring of macroporous silicon for applications as photonic crystals $J$. Porous Mat. 7 201-4

[15] Teo E J, Breese M B, Bettiol A A, Mangaiyarkarasi D, Champeaux F, Watt F and Blackwood D J 2006 Multicolor photoluminescence from porous silicon using focused, highenergy helium ions Adv. Mater. 18 51-5

[16] Dang Z, Breese M B, Recio-Sánchez G, Azimi S, Song J, Liang H and Martín-Palma R J 2012 Silicon-based photonic crystals fabricated using proton beam writing combined with electrochemical etching method Nanoscale Res. Lett. 7416

[17] Recio-Sánchez G, Dang Z, Torres-Costa V, Breese M B and Martín-Palma R J 2012 Highly flexible method for the fabrication of photonic crystal slabs based on the selective formation of porous silicon Nanoscale Res. Lett. 7449

[18] Punzón-Quijorna E, Sánchez-Vaquero V, Muñoz-Noval Á, Pérez-Roldán M J, Martín-Palma R J, Rossi F and TorresCosta V 2012 Nanostructured porous silicon micropatterns as a tool for substrate-conditioned cell research Nanoscale Res. Lett. 7396

[19] Torres-Costa V, Martínez-Muñoz G, Sánchez-Vaquero V, Muñoz-Noval Á, González-Méndez L, Punzón-Quijorna E and Martín-Palma R J 2012 Engineering of silicon surfaces at the micro-and nanoscales for cell adhesion and migration control Int. J. Nanomed. 7623

[20] Buriak J M 2006 High surface area silicon materials: fundamentals and new technology Phil. Trans. R. Soc. A $364217-25$

[21] Naveas N, Costa V T, Gallach D, Hernandez-Montelongo J, Palma R J M, Garcia-Ruiz J P and Manso-Silván M 2012 Chemical stabilization of porous silicon for enhanced biofunctionalization with immunoglobulin Sci. Technol. Adv. Mater. 13045009

[22] Huster J, Müller J, Renner H and Brinkmeyer E 2011 Scanning phase-mask DUV inscription of short-period large-area photoresist gratings J. Lightwave Technol. 29 2621-8

[23] Torres-Costa V, Martín-Palma R J and Martínez-Duart J 2004 Optical constants of porous silicon films and multilayers determined by genetic algorithms J. Appl. Phys. 96 4197-203

[24] Vega F, Peláez R J, Kuhn T, Afonso C N, Recio-Sánchez G and Martín-Palma R J 2014 Ultraviolet laser patterning of porous silicon J. Appl. Phys. 115184902

[25] Shen Q and Toyoda T 2003 Dependence of thermal conductivity of porous silicon on porosity characterized by photoacoustic technique Rev. Sci. Instrum. 74 601-3

[26] Low S P, Voelcker N H, Canham L T and Williams K A 2009 The biocompatibility of porous silicon in tissues of the eye Biomaterials 30 2873-80

[27] Arroyo-Hernandez M, Martín R J, Perez-Rigueiro J, García J P, García J L and Martínez-Duart J M 2003 Biofunctionalization of surfaces of nanostructured porous silicon Mater. Sci. Eng. C 23 697-701

[28] Majoul N, Aouida S and Bessaïs B 2015 Progress of porous silicon APTES-functionalization by FTIR investigations Appl. Surf. Sci. 331 388-91

[29] Khung Y L, Graney S D and Voelcker N H 2006 Micropatterning of porous silicon films by direct laser writing Biotechnol. Prog. 22 1388-93 